\title{
Research Paper: Comparing Short Clinical Evaluation Exercise and Direct Observation of Procedural Skills With the Traditional Evaluation Approach on the Clinical Skills of Forensic Medicine Residents
}

\author{
Fares Najari ${ }^{*}$ (D), Dorsa Najari ${ }^{1}$ (D)
}

1. Department of Forensic Medicine, School of Medicine, Shahid Beheshti University of Medical Sciences, Tehran, Iran.

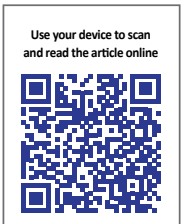

ditation: Najari F, Najari D. Comparing Short Clinical Evaluation Exercise and Direct Observation of Procedural Skills With the Traditional Evaluation Approach on the Clinical Skills of Forensic Medicine Residents. International Journal of Medical Toxicology and Forensic Medicine. 2020; 10(1):27118. https://doi.org/10.32598/ijmtfm.v10i1.27118

ointtps://doi.org/10.32598/ijmtfm.v10i1.27118

Article info:

Received: 16 Sep 2019

First Revision: 27 Sep 2019

Accepted: 05 Nov 2019

Published: 03 Apr 2020

\section{Keywords:}

Clinical evaluation, Short clinical evaluation exercise (Mini-CEX), Direct observation of procedural skills (DOPS), Forensic medicine

\section{A B STRACT}

Background: The development of clinical teaching necessitates the use of novel and appropriate clinical evaluation methods. In the meantime, the use of new evaluation approaches that enhance learning at the same time has been recommended. This study aimed to compare the effect of the two new evaluation methods of direct observation of procedural skills (DOPS) and short clinical evaluation exercise (Mini-CEX) with the conventional evaluation method on clinical skills of forensic medicine residents.

Methods: This is a randomized trial performed among forensic medicine residents. Using Cochran's formula, the minimum sample size was calculated to be 25 individuals per group. All the residents were randomly divided into either the experimental or the control groups. After training and evaluation, the experimental group was tested using the DOPS and MiniCEX methods three times during the course, and the control group was evaluated using the conventional method. The basis for the evaluation of the procedures (physical examination and autopsy) in both groups was the valid and reliable checklists prepared by the researchers. The results were analyzed using descriptive and inferential statistics (e.g., the Chi-square and independent t-test).

Results: There was no significant difference between the two groups in terms of demographic variables such as age, sex, and grade point average (GPA) $(\mathrm{P}>0.06)$. The means of total scores were significantly higher in the experimental group (using the Mini-CEX and DOPS methods) than the control group (conventional method) $(\mathrm{P}<0.0002)$.

Conclusion: The use of new evaluation methods of DOPS and Mini-CEX improved the clinical skills of forensic medicine residents. Therefore, it is recommended that resident training centers use these two methods of assessment, together with other methods, to evaluate clinical procedures and boost forensic residents' learning.

\footnotetext{
* Corresponding Author:

Fares Najari, MD.

Address: Department of Forensic Medicine, School of Medicine, Shahid Beheshti University of Medical Sciences, Tehran, Iran. Tel: +98 (21) 23872540

E-mail:najari.hospital@sbmu.ac.ir
} 


\section{Introduction}

A

11 forensic medicine residency curricula are dedicated to clinical education, and one of the critical and challenging issues in clinical education is residents' educational evaluation in the field. Evaluation is one of the most important tasks of a clinical professor. With proper evaluation, we can identify the strengths and weaknesses of education, and by reinforcing the positive aspects and removing the flaws, we can take a step toward developing and reforming the educational system $[1,2]$. Effective evaluation not only motivates forensic medical residents but also assists instructors in evaluating their performance. Combined with feedback, it can be useful in enhancing learning skills. Because of changes in clinical education approaches, the necessity of using new assessment methods is becoming increasingly clear [3].

In a study conducted in a school of nursing in the southern states of America, it was found that the clinical evaluation methods were not revised in $45 \%$ of colleges for 5 years, $35 \%$ of colleges for $6-10$ years, $17 \%$ of colleges for $11-15$ years, and 3\% of colleges for more than 15 years [4]. Also, a study in Tehran nursing faculties reported that $62 \%$ of nursing students believed that clinical evaluation factors and conditions were not satisfactory for all nursing students [5].

Experts have been searching for valid methods for years to evaluate students' clinical performance effectively. Evaluation methods with their feedbacks, and evaluating complex cases in the traditional assessment of students, have also promoted learning. For evaluation in the clinical setting, different methods such as performance observation, 360-degree evaluation, simulated clinical test, task set method, objective structured clinical examination (OSCE), short clinical evaluation exercise (Mini-CEX), and direct observation of procedural skills (DOPS) are used [6, 7].

DOPS is a method for evaluating and providing feedback on practical skills. In this method, the observation and evaluation of clinical skills are performed by the resident on a real patient, and in each procedure, the evaluator is different. Each test is conducted on an essential practical skill. Each test is held 4-6 times a year. Venipuncture, intravenous injection, peripheral and central venous blood sampling, electrocardiography, and arterial blood sampling are among the procedures that can be evaluated by DOPS $[8,9]$.
Another essential way to assess the clinical skills of students or residents is Mini-CEX. It is a test for evaluating clinical skills and giving feedback on performance at the same time. The instructor observes the trainee while operating, examining the body, taking history from the patient or the relatives, and reporting his or her diagnosis and plan. Applications of this method include history taking, physical examination, professional behavior, clinical judgment, communication skills, organization, and efficiency $[10,11]$.

Both Mini-CEX and DOPS methods for each procedure are run in three steps with a specified time interval. At the end of each step, the evaluator observes the student or resident during the procedure and gives feedback to the student or resident and points out the strengths and weaknesses of the trainee. At the end of the third stage of evaluation, the evaluator determines the student's or resident's performance score using a structured form and then gives feedback $[12,13]$. It usually takes about 15 minutes for the test and 5 minutes for feedback. All the skills in the form are scored based on a 6-point scale. According to the results of the research, the usual evaluation of residents or students is limited to subjective information and lacks careful evaluation of their clinical skills $[14,15]$.

The results of the study showed that $62 \%$ of male students and $82 \%$ of female students believed that all skills could not be evaluated through conventional evaluation, and this dissatisfaction can be a deterrent to learning [16].

No studies have been published in Iran to investigate the impact of the implementation of the DOPS and MiniCEX methods on the assessment of clinical skills of forensic medicine residents. Because of the limitations of conventional evaluation methods and lack of literature on the impact of novel evaluation methods on residents' clinical skills, the present study aimed to compare the effect of the routine evaluation method with the two new evaluation methods of DOPS and Mini-CEX on the clinical skills of forensic medical residents in Tehran, Iran.

\section{Materials and Methods}

This study was designed as a randomized trial after obtaining permission from the Legal Medicine Organization of Tehran Province. All forensic medicine residents of three medical sciences universities of Tehran, Iran (e.g., Tehran, Iran, and Shahid Beheshti) from 4 years ago who were taking autopsy courses were randomly assigned to the control or experimental groups $(\mathrm{n}=25)$. 
Five clinical instructors (forensic specialists working in the autopsy room) were also randomly assigned to the experimental and control groups. Forensic medical residents and instructors were allowed to withdraw from the study at any time when they did not wish to continue. The procedures used in this study were as follows: Mini-CEX method for evaluating skills (i.e., cadaveric examination report, history-taking from relatives, intern training) and DOPS method for assessing practical skills (i.e., cadaveric peripheral vein blood sampling, cadaver appearance examination, bone and skin incision, and internal organs examination).

The study had two general phases: a) preparation of tools and training of trainers and b) implementation of the evaluation program. In the first phase, before the evaluation, the required training protocol for these two new methods was provided, and the experimental group instructors were trained during a 2-hour workshop.

In the second phase, forensic medicine residents in the control group were evaluated based on the conventional method of forensic autopsy in Tehran Province, and the experimental group was evaluated based on the DOPS and Min-CEX methods. In the traditional method, the resident's skill is usually subjectively judged by the instructor during the general skill training course, and it is scored accordingly. In the experimental group, the clinical performance of forensic medicine residents was evaluated using the checklist-based evaluation method. The evaluation steps in the experimental group were as follows:

A) First stage: observing skills in 15 minutes and giving feedback in 5 minutes

B) Second stage: repeating the first-stage after two weeks (emphasis on the resident's strengths and weaknesses)

C) Third stage: repeating the first stage after four weeks and give the final score to the control group.

Six procedures were evaluated in only one step. The final evaluation of the forensic medicine residents in both groups was performed based on an already prepared checklist by the researchers. A score of zero means that the resident did not perform appropriately for the procedure; other scores were as follows: 1) lower than expected, 2) borderline, 3) as expected, 4) higher than expected.
To evaluate the psychometric properties of the checklists, we performed an extensive library, and electronic search on how each procedure was performed, and an initial draft of the checklist was prepared for each procedure. The draft was then given to ten forensic faculty members from Shahid Beheshti, Iran, and Tehran universities of medical sciences.

After applying expert opinions, the final tools were prepared under the supervision of the executor. Ten experts approved the content validity of the tools, and Lawshe's minimum content validity ratio (CVR) was 0.64 . On this basis, the items obtaining the required score were chosen. The content validity index (CVI) of above 0.75 was considered appropriate for the questions. If CVI was $0.65-0.75$, the questions were revised by the experts, and the questions that scored less than 0.65 were not accepted.

In this study, inter-rater reliability and equivalence methods were used to determine the reliability of the evaluators. Three instructors observed and evaluated at least five residents for each procedure simultaneously. The intra-cluster correlation coefficient (ICC) was used to evaluate the agreement among evaluators. For each item, the kappa test was used (the kappa coefficient was 0.5 , and the ICC was 4.0). The Chi-square test, independent $t$ test, and Pearson correlation were employed according to the type of variables studied.

\section{Results}

In the study of homogeneity of the two groups in terms of age and grade point average (GPA) of the general medical course, the independent $t$ test was run. Based on the Chi-square test, no significant difference between the two groups was noted in terms of their gender and marital status. Tables 1 and 2 present the descriptive results of the demographic variables of the two groups.

Table 3 presents the results of comparing the final evaluation scores of the experimental and control groups. According to the data in Table 3, the independent $t$ test shows a significant difference between the scores of the two evaluation procedures of the two groups. The correlation of the GPA and age of the forensic residents with the scores of the six clinical procedures was determined using the Pearson coefficient. This test showed that the mean scores of sampling of peripheral veins, cadaveric appearance, skin and bone incisions, internal organs examination $(\mathrm{P}<0.002, \mathrm{r}=0.24)$, cadaver examination reporting, relatives history-taking and intern training 
Table 1. Frequency distribution of quantitative demographic variables based on the study groups

\begin{tabular}{|c|c|c|c|c|c|}
\hline \multirow{3}{*}{ Variables } & \multicolumn{2}{|c|}{ Study Groups } & \multicolumn{3}{|c|}{ Statistical Results } \\
\hline & \multicolumn{2}{|c|}{ Mean士SD } & \multirow{2}{*}{ df } & \multirow{2}{*}{$\mathbf{t}$} & \multirow{2}{*}{$\mathbf{P}$} \\
\hline & Experimental Group & Control Group & & & \\
\hline Age $(y)$ & $34 \pm 0.84$ & $33.8 \pm 0.58$ & 68 & -9.273 & $>0.62$ \\
\hline GPA (grade point average) & $15.9 \pm 1.59$ & $16.19 \pm 1.42$ & 68 & -0.752 & $>0.524$ \\
\hline
\end{tabular}

Table 2. Absolute (and relative) distribution of nominal demographic variables based on the study groups

\begin{tabular}{|c|c|c|c|c|}
\hline \multirow{2}{*}{ Variables } & \multirow{2}{*}{ Characteristics } & \multicolumn{2}{|c|}{ No. (\%) } & \multirow{2}{*}{ Statistical test } \\
\hline & & Experimental group & Control group & \\
\hline \multirow{2}{*}{ Marital status } & Married & $10(31.4)$ & $12(48)$ & $\chi^{2}=1.5$ \\
\hline & Single & $15(68.6)$ & $13(52)$ & $\mathrm{P}=0.22$ \\
\hline \multirow{2}{*}{ Gender } & Female & $13(52)$ & $19(60)$ & $\chi^{2}=232$ \\
\hline & Male & $12(48)$ & $11(40)$ & $P=0.62$ \\
\hline
\end{tabular}

Table 3. Comparing the mean and standard deviation of the final scores of the procedures in the experimental and control groups using the Independent t-test

\begin{tabular}{|c|c|c|c|c|c|}
\hline \multirow{2}{*}{ Procedure Group } & \multicolumn{2}{|c|}{ Mean $\pm S D$} & \multirow{2}{*}{$\mathbf{t}$} & \multirow{2}{*}{ df } & \multirow{2}{*}{$\mathbf{P}$} \\
\hline & Experimental & Control & & & \\
\hline Cadaveric examination reporting & $130.77 \pm 10.92$ & $130.77 \pm 13.42$ & -10.081 & 68 & 0.002 \\
\hline Trainee training & $61.25 \pm 7.25$ & $49.17 \pm 6.34$ & -6.218 & 68 & 0.001 \\
\hline External examination during autopsy & $95.24 \pm 4.35$ & $51.23 \pm 3.42$ & -10.435 & 68 & 0.004 \\
\hline Sampling of peripheral/vitreous vessels & $11.34 \pm 10.24$ & $86.08 \pm 10.92$ & -10.373 & 68 & 0.003 \\
\hline Skin and bone incision & $94.22 \pm 8.15$ & $66.62 \pm 9.82$ & -11.858 & 68 & 0.001 \\
\hline Examination of internal organs of the body & $65.45 \pm 7.54$ & $32.25 \pm 5.31$ & -11.254 & 68 & 0.003 \\
\hline
\end{tabular}

$(\mathrm{P}=0.001, \mathrm{r}=0.35)$ were significantly correlated with the GPA and age of residents.

\section{Discussion}

This study aimed to compare the effects of new evaluation methods with the routine method on the clinical skills status of forensic medicine residents. The results of this study showed that the Mini-CEX and DOPS tests, compared to the traditional evaluation method, have a more significant impact on enhancing the skills of forensic residents.

In a study to evaluate the effect of DOPS test on clinical skills of medical interns in the Gynecology Department of Kurdistan University of Medical Sciences, Shah-Gheibi et al. evaluated seven skills using the DOPS method. In their study, it was found that DOPS use was effective in improving the skills of medical students $[17,18]$. 
Another study by Parner et al. demonstrated that Mini-CEX could be incorporated into the surgical internship course. Also, this study emphasized that the content of the feedback makes the Mini-CEX a rich evaluation tool and is very valuable in terms of critical and supportive feedback [19].

Concerning the successful results in the present study, the Mini-CEX could serve as a vital tool for the clinical evaluation of the residents. Investigating the relationship between residents' GPA and mean age and their clinical skills scores of sampling of peripheral/vitreous vessels, cadaver appearance examination, bone and skin incision, examination of internal organs, reporting cadaveric examination, history-taking from relatives and intern training reflected significant results, and those with higher GPAs obtaining better scores in these six procedures. Also, younger interns attained better scores in this regard. In the study of Noohi et al., there was a significant correlation between the average scores of the previous semesters and the mean of clinical skills in the special wards, such that students with higher average scores had higher proficiency in the special wards [20]. Given that no significant relationship was observed in the other two procedures in the present study, the residents' clinical proficiency level is directly related to their GPA and age. Further studies with larger sample sizes should be performed to investigate this relationship.

In a study performed by Wiles et al., it was concluded that male medical students and those with poor academic performance might not use feedback to evaluate their learning experiences [21]. The results of Chehrzad et al. study in Guilan showed that $87.6 \%$ of students obtained acceptable scores using DOPS. Also, the students' scores on the DOPS test had a normal distribution. Finally, they concluded that DOPS is a useful tool for evaluating students' skills.

In the present study, there was an upward trend in the scores of procedures evaluated by DOPS. The limitations of this study include the low number of forensic medical residents across the country and Tehran and the unwillingness of some instructors to use these novel evaluation methods. Besides, due to the nature of the study, performing a pretest was not feasible.

\section{Conclusion}

In general, the results of this study showed a significant difference between the final scores of the Mini-CEX and DOPS tests and the traditional evaluation test. Thus, because of the nature of the methods, they have a more significant impact on improving the skills of forensic medicine residents than traditional evaluation methods. In conclusion, further attention should be paid to new evaluation methods, and these procedures should be more applied in evaluating forensic medical residents to reach the final goal, i.e., educating forensic specialists.

\section{Ethical Considerations}

\section{Compliance with ethical guidelines}

Information obtained from patients' records was considered confidential. The Etical Code number from the Medical Ethics Committee of Shahid Beheshti University of Medical Sciences is $1398 / 68555$.

\section{Funding}

This research did not receive any specific grant from funding agencies in the public, commercial, or not-forprofit sectors.

\section{Author's contributions}

All authors contributed to designing, running, and writing all parts of the research.

\section{Conflict of interest}

The authors declared no conflict of interest.

\section{Acknowledgements}

We wish to thank the officials of the forensic mortuary of Tehran's Legal Medicine Center, and the forensic specialists and interns who contributed to carrying out this study.

\section{References}

[1] Moiz B, Ali SK, Rashid A, Shariq M, Karim F. Development and pilot testing of a novel tool for evaluating practical skills in hematopathology residents in Pakistan. Journal of Graduate Medical Education. 2019; 11(Suppl 4):177-80. [DOI:10.4300/JGME-D-18-00361].

[2] Lörwaldz AC, Lahner FM, Mooser B, Perrig M, Widmer MK, Greif R, et al. Influences on the implementation of MiniCEX and DOPS for postgraduate medical trainees' learning: A grounded theory study. Medical Teacher. 2019; 41(4):44856. [DOI:10.1080/0142159X.2018.1497784] [PMID]

[3] Kipen E, Flynn E, Woodward-Kron R. Self-regulated learning lens on trainee perceptions of the Mini-CEX: A qualitative 
study. British Medical Journal (BMJ Open). 2019; 9(5):e026796 [DOI:10.1136/bmjopen-2018-026796] [PMID] [PMCID]

[4] Wei CJ, Lu TH, Chien SC, Huang WT, Liu YP, Chan CY, et al. The development and use of a pharmacist-specific MiniCEX for postgraduate year trainees in Taiwan. BMC Medical Education. 2019; 22:19(1):165. [DOI:10.1186/s12909-019-16022] [PMID] [PMCID]

[5] Mortaz Hejri S, Jalili M, Masoomi R, Shirazi M, Nedjat S, Norcini J. The utility of mini-clinical evaluation exercise in undergraduate and postgraduate medical education: A BEME review: BEME Guide No. 59. Medical Teacher. 2020; 42(2):125-42. [DOI:10.1080/0142159X.2019.1652732] [PMID]

[6] Bansal M. Introduction of directly observed procedural skills (DOPS) as a part of competency-based medical education in otorhinolaryngologyIndian. Journal of Otolaryngology and Head \& Neck Surgery. 2019; 71(2):161-6. [DOI:10.1007/ s12070-019-01624-y] [PMID]

[7] Rafii F, Ghezeljeh TN, Nasrollah S. Design and implementation of clinical competency evaluation system for nursing students in medical-surgical wards. Journal of Family Medicine and Primary Care. 2019; 8(4):1408-13. [DOI:10.4103/jfmpc. jfmpc_47_19] [PMID] [PMCID]

[8] Ali L, Ali S, Orakzai N, Ali N. Effectiveness of direct observation of procedural skills (DOPS) in postgraduate training in urology at institute of kidney diseases, Peshawar. Journal of the College of Physicians and Surgeons. 2019; 29(6):516-19. [DOI:10.29271/jcpsp.2019.06.516] [PMID]

[9] Siau K, Crossley J, Dunckley P, Johnson G, Feeney M, Hawkes ND, et al. Direct observation of procedural skills (DOPS) assessment in diagnostic gastroscopy: Nationwide evidence of validity and competency development during training. Surgical Endoscopy. 2020; 34(1):105-14. [DOI:10.1007/s00464019-06737-7] [PMID] [PMCID]

[10] Soemantri D, Dodds A, Mccoll G. Examining the nature of feedback within the Mini Clinical Evaluation Exercise (Mini-CEX): an analysis of 1427 Mini-CEX assessment forms. GMS Journal for Medical Education. 2018; 15:35(4):Doc47. [DOI:10.3205/zma001193eCollectio 2018.] [PMID].

[11] Berendonk C, Rogausch A, Gemperli A, Himmel W. Variability and dimensionality of students and supervisors' MiniCEX scores in undergraduate medical clerkships: A multilevel factor analysis. BMC Medical Education. 2018; 18(1):100. [DOI:10.1186/s12909-018-1207-1] [PMID] [PMCID]

[12] Lörwald AC, Lahner FM, Greif R, Berendonk C, Norcini J, Huwendiek S. Factors influencing the educational impact of Mini-CEX and DOPS: A qualitative synthesis. Med Teach. 2018; 40(4):414-20. [DOI:10.1080/0142159X.2017.1408901] [PMID]

[13] Atash Sokhan G , Bolbol Haghighi N, Bagheri H, Ebrahimi $\mathrm{H}$. [Comparison of self, peer, and clinical teacher evaluation in clinical skills evaluation process of midwifery students (Persian)]. Iranian Journal of Medical Education. 2011; 10(4):333-9.

[14] Rahimi A, Ahmadi F. The obstacles and improving strategies of clinical education from the viewpoints of clinical instructors in Tehran's Nursing Schools (Persian)]. Iranian Journal of Medical Education. 2005; 5(2):73-80.
[15] Zareiyan Jahromi A, Ahmadi F. Learning needs assessment in bachelor of nursing: a qualitative research (Persian)] Iranian Journal of Medical Education. 2005; 5(2):81-92.

[16] Smith-Strom H, Nortvedt MW. Evaluation of evidencebased methods used to teach nursing students to critically appraise evidence. Journal of Nursing Education. 2008; 47(8):372-5. [DOI:10.3928/01484834-20080801-08] [PMID]

[17] Shahgheibi Sh, Pooladi A, BahramRezaie M, Farhadifar F, Khatibi R. Evaluation of the effects of Direct Observation of Procedural Skills (DOPS) on clinical externship students' learning level in obstetrics ward of Kurdistan University of Medical Sciences. Journal of Medicine Eduction. 2009; 13(12):29-33.

[18] Noohi E, Motasedi M, Haghdoost A. [Clinical teachers' viewpoints towards objective structured clinical examination in Kerman University of Medical Science (Persian)]. Iranian Journal of Medical Education. 2008; 8(1):113-9.

[19] Castanelli DJ, Moonen-van Loon JMW, Jolly B, Weller JM. The reliability of a portfolio of workplace-based assessments in anesthesia training. Canadian Journal of Anaesthesia. 2019; 66(2):193-200. [DOI:10.1007/s12630-018-1251-7] [PMID]

[20] Wiles CM, Dawson K, Hughes TA, Llewelyn JG, Morris $\mathrm{HR}$, Pickersgill TP, et al. Clinical skills evaluation of trainees in a neurology department. Clinical Medicine. 2007; 7(4):3659. [DOI:10.7861/clinmedicine.7-4-365] [PMID] [PMCID]

[21] Chehrzad M , Sohail SZ, Mirzaee M, Kazem Nejad E. [Compare of Osce and traditional clinical evaluation methods on nursing students' satisfaction (Persian)]. Journal of Medical Faculty Guilan University of Medical Sciences. 2007; 13(50):8-12. 